\title{
Modification of preference in a concurrent schedule by aversive conditioning: An analog study
}

\author{
VERNON L. QUINSEY and GEORGE W. VARNEY \\ Mental Health Center, Penetanguishene, Ontario, Canada LOK 1P0
}

\begin{abstract}
A difficulty with explanations of aversion therapy which rely on the conditioned emotional response (CER) paradigm is that the response rates of humans are not very sensitive to the CER procedure. This study examined how a CER procedure affected choice. Eight male volunteers were first exposed to a two-component concurrent random interval schedule in which a green light was associated with the richer component, and a red with the leaner component. Then the green light was made the $\mathrm{CS}^{+}$and the red light the CS - in a differential CER procedure using shock as the US. Finally, the concurrent schedule was reinstated to determine whether the relative preference for the two components had been altered by the CER procedure. Little alteration in preference was observed.
\end{abstract}

Aversion therapy is one of the most widely used treatments in the modification of inappropriate sexual preference. Aversion therapy techniques have usually been inspired by aversive conditioning paradigms and theoretical accounts used in the classical and operant animal-conditioning literature. One of the most influential interpretations of changes in sexual preference that occur as a result of aversion therapy has been provided by Rachman and Teasdale (1969). These authors have argued that aversion therapy works by classically conditioning anxiety to stimuli associated with deviant behavior; the anxiety then subtracts from the incentive motivation to engage in the deviant behavior.

The theory, therefore, asserts that deviant instrumental sexual behavior is reduced by the classical aversive conditioning component of aversion therapy. A problem with this theoretical account (in addition to those outlined by Hallam, Rachman, \& Falkowski, 1972) is that it is very difficult to obtain reliable suppression of operant behavior in human subjects with classical conditioning techniques. From the theory, it would be expected that large amounts of conditioned suppression of arbitrarily defined operants could be found in the laboratory within individual subjects using standard conditioned emotional response procedures (e.g., Ayres \& Quinsey, 1970). However, Sachs and Keller (1972) have shown that short interstimulus intervals and high shock intensities are required to obtain conditioned suppression in human subjects. The conditional stimulus and shock parameters typically employed in aversion therapy treatments (e.g., Rachman \&

This research was supported by Grants OMHF 447-73B and C, and OMHF 487-74A to the first author. Dr. J. Ayres made helpful comments on an earlier version of this paper.
Teasdale, 1969) do not appear likely to produce conditioned suppression of operant behavior.

It may be, however, that the laboratory studies of conditioned suppression are not good analogs of the aversion therapy procedure because, in the laboratory situation, a reduction in absolute response rate is examined whereas in the aversion therapy situation a shift in sexual preferences is produced. An operant choice measure may, therefore, be a better analog of the relevant parameters in aversion therapy than a frequency measure. The purpose of the present study was to determine whether an aversive conditioning procedure produced large changes in preferences exhibited by human subjects responding on a concurrent schedule.

\section{EXPERIMENT I}

\section{Method}

Subjects. Four male volunteer high school students, aged 17, 16, 15 , and 16 years participated.

Apparatus. Subjects sat in a small room isolated from the electromechanical programming apparatus and faced a $50 \times 80 \mathrm{~cm}$ wooden panel. The panel contained one Lindsley manipulandum on the left side and one Lindsley manipulandum on the right side. A bank of red lights were on the left side and a bank of green lights were on the right side of a Lehigh Valley 521-66 light panel located in the upper middle area of the panel. The center of the panel contained a point counter (Lehigh Valley 521-76) with a red light on the left side and a green light on the right side.

Procedure. Subjects were exposed in sequence to two differential reinforcement of low rates (DRL) sessions, four concurrent random interval (RI) sessions, one aversive conditioning session, and two additional concurrent $\mathrm{RI}$ sessions.

Subjects were first exposed to the DRL sessions because our pilot work had shown that subjects exhibited little preference acquisition in concurrent schedules, and we obtained suggestive evidence that pre-exposure to a DRL schedule would improve this situation. The DRL sessions were 30 min long. Reinforcement was set up to follow the first response that occurred after every response-free 10-sec interval; each response reset the 10 -sec timer. Both the red and 


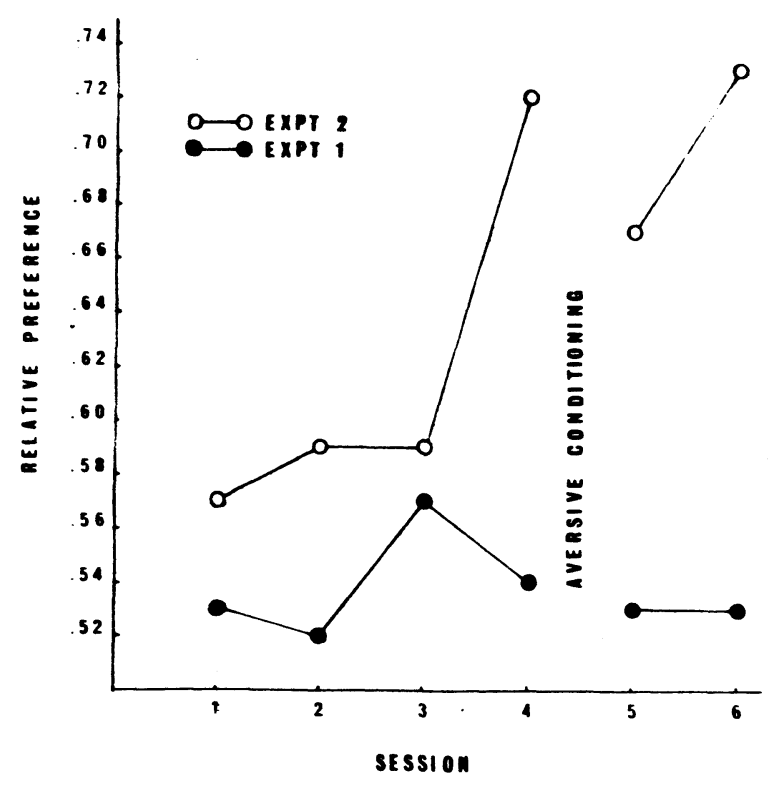

Figure 1. Relative preference for the richer (green) component of the concurrent schedule for both experiments.

green banks of lights were illuminated during the session. The red and green lights beside the counter flashed each time a point was earned. For the DRL sessions, subjects were instructed that they could sometimes earn points by pulling the lever on their right, that their task was to earn as many points as possible, and that they would earn one cent for each point they earned. Watches were removed before all sessions.

Before the concurrent schedule sessions, the subjects were instructed as follows: "When the session starts, either the red or green lights will come on. You may sometimes change these lights by pulling this lever (the experimenter indicates the left lever). During the session, points will sometimes be available to you, and it is your task to get as many of them as you can. You will sometimes be rewarded for pulling this lever (the experimenter indicates right lever). Any points you earn will be shown on this counter. Either a red or a green light will flash beside the counter when you earn a point. You will be paid three cents for each point you make. This session will last $30 \mathrm{~min}$. Do you understand the instructions?"

The concurrent RI schedule was programmed by feeding impulses into two probability generators from a VI 30 -sec tape (Fleshler \& Hoffman, 1962). The probability generators were set at .6 and .15 for the green and red components, respectively. Pulses from the probability generators set up reinforcement for responses on the right lever in the green component, on the average, every $50 \mathrm{sec}$ and in the red, on the average, every $200 \mathrm{sec}$. Thus the discriminative stimulus for the richer schedule was the illumination of the green lights on the right side, and the cue associated with the leaner RI schedule was the illumination of the red lights on the left side. Responses on the changeover (left) lever were followed by an unsignaled time-out from reinforcement of $5 \mathrm{sec}$.

The aversive conditioning session was similar to the aversion therapy procedure of Quinsey, Bergersen, \& Steinman (Note 1). Subjects were told that we were interested in the subjective effects of shock and were given some rating tasks after the session. They were instructed to watch the banks of lights and that they would receive one dollar for the 20-min session. One subject terminated the aversive conditioning session after $10 \mathrm{~min}$. Subjects were not required to pull the lever, and no point reinforcements were available. Shock electrodes were attached to the inner surface of the upper left arm. In preparation for the aversive conditioning session, the intensity of brief manually delivered shocks was increased until the shock was judged to be painful and as high as the subject wished to tolerate. The $\mathrm{CS}^{+}$was an illumination of the green bank of lights (the $S \mathbf{D}$ for the richer of the components in the concurrent schedule) and the CS- was an illumination of the red lights. The conditional stimuli were $10 \mathrm{sec}$ in duration and separated by a variable intertrial interval of $18 \mathrm{sec}(10,20$, or $30 \mathrm{sec})$. A 2 -sec shock occurred in the last $2 \mathrm{sec}$ of a randomly selected $80 \%$ of the $\mathrm{CS}^{+}$presentations. There were 20 presentations of each of the two stimuli which alternated in a fixed irregular sequence. Following the aversive conditioning session, subjects were re-exposed to the concurrent schedule.

\section{Results and Discussion}

The preference data are shown in Figure 1. All subjects spent slightly more time in the presence of the green $\mathrm{SD}$ during the last two concurrent sessions before the aversive conditioning analog session. In the two concurrent sessions following aversive conditioning, three out of four subjects showed slight preference for the green schedule. None of the changes were large relative to baseline variability. In the first $\mathbf{1 5}$ min of the first postaversive conditioning session, all subjects spent more than $50 \%$ of the time in the green schedule.

In general, relative response rates in the concurrent RI schedule paralleled the relative time the subjects spent in the two components but, because there was more within-subject variability in the response rate data, they are not presented here. RI response rates were very low (because of the DRL sessions) and decreased somewhat after the aversive conditioning session. There was an average of 5.49 responses per minutes (averaged over subjects) in the four sessions before aversive conditioning and 3.71 in the two postaversive conditioning sessions. There was an average of 4.75 changeovers per minute before aversion and 3.82 after.

There was, therefore, no evidence for a shift in preference as a result of the "off-the-baseline" conditioned emotional response procedure. It cannot be argued that the aversion session was too brief to produce a change in preference because studies in the animal literature routinely produce conditioned suppression effects with fewer than $16 \mathrm{CS}^{+}$-shock pairings. There is, in fact, evidence that most conditioned suppression effects occur with the first few pairings (e.g., Benedict \& Ayres, 1972).

It may be objected that significant alterations of preference may have been obtained by running additional subjects. Aversion therapy is, however, expected to produce alterations in sexual preference within individual subjects. An aversive conditioning interpretation of aversion therapy requires that large changes in behavior be produced within individual subjects by CER techniques.

A more serious difficulty, however, is the lack of clear preference acquisition in any of the subjects. Since the subjects spent approximately half of their time in the richer component in all the concurrent RI sessions despite the large differences in reinforcement 
frequency between the two components, it can well be argued that this study has demonstrated that the concurrent procedure is merely insensitive to any experimental manipulation.

\section{EXPERIMENT II}

The purpose of the second experiment was to alter the concurrent procedure so as to produce a preference for the richer component, thereby providing an opportunity to perform a more convincing assessment of preference shifts associated with the CER procedure. Because it has been found that lowering the number of changeover responses can make human subject's concurrent performance more orderly (Baum, 1975), and subjects in Experiment I exhibited a high changeover rate, it was decided to attempt to lower the number of changeovers by making point loss contingent on them. In this experiment, therefore, a point was subtracted from the subject's panel for every fourth changeover.

\section{Method}

Subjects. Four male volunteers, high school students aged 18, 17, 17 , and 17 years served as subjects.

Apparatus and procedure. The apparatus and procedure were the same as in Experiment I with the following alterations: The subjects were not pre-exposed to the DRL schedule. At the start of the concurrent sessions, the subject's point counter was set at 50 . Each point was worth two cents. Subjects were told that a point would occasionally be subtracted from their total when they pulled the changeover lever. The instructions were altered to accommodate these changes. One point was subtracted from the subject's panel for changeovers on a fixed-ratio 4 schedule.

Changeover responses, response rate, and relative time spent in the preferred schedule were compared between experiments for the last pre-CER session with one-tailed Wilcoxon rank sum tests. The probability and $T$ values reported below refer to these comparisons.

\section{Results and Discussion}

There was an average of 68.9 responses per minute on the reinforcement lever in Session 4 of Experiment II. This response rate was higher than that found in the first experiment $(T=10, p<.025)$ presumably because of the pre-exposure of the first four subjects to the DRL schedule. In contrast, there were fewer changeovers per minute in the second study than in the first. A mean of 4.24 in the fourth session of Experiment $I$ and a mean of .82 in Experiment II $(\mathrm{T}=10, \mathrm{p}<.025)$.

Three of the four subjects in Study 2 showed unequivocal acquisition of a preference for the richer $\mathrm{RI}$ component over the first four sessions. The average relative time allocation data are shown in Figure 1. Subjects in Experiment II showed greater preference for the richer schedule than those in Experiment I $(\mathrm{T}=11.5, \mathrm{p}<.05)$. The procedure of charging for changeovers apparently facilitated preference acquisition. The hypothesis that pre-exposure to a DRL schedule hinders preference acquisition can be discounted on the basis of our pilot work.
As in the first experiment, the CER procedure had little effect. One subject increased the proportion of time he spent in the green stimulus and three subjects showed the reverse effect. As can be seen in the figure, these changes were small. The choice measure used with a changeover cost was, therefore, sensitive to differences in reinforcement frequency but not to the CER procedure.

The difference between concurrent Sessions 4 and 5 was evaluated with a Wilcoxon signed ranks test for the relative time allocation data from both experiments. The difference was not significant $(T=13, p>.05)$. The data from both experiments, therefore, do not support a conditioned emotional response interpretation of aversion therapy because changes in preference in the concurrent schedule were not found to be produced by an aversive conditioning procedure similar to those employed in aversion therapy. The results are in agreement with nonclinical laboratory studies in indicating that conditioned suppression of arbitrarily chosen operants is difficult to obtain with human subjects. The failure to demonstrate large preference changes within individual subjects in this analog of aversion therapy suggests that theoretical accounts of aversion therapy should employ explanatory principles that do not involve classical conditioning of fear responses.

\section{REFERENCE NOTE}

1. Quinsey, V. L., Bergersen, S. G., \& Steinman, C. M. Changes in physiological and verbal responses of child molesters during aversion therapy. Paper presented at the meeting of the Canadian Psychological Association in Quebec City, June 1975.

\section{REFERENCES}

Ayres, J. J. B., \& Quinsey, V. L. Between-groups incentive effects on conditioned suppression. Psychonomic Science, 1970, 21. 294-296.

BAUM, W. M. Time allocation in human vigilance. Journal of the Experimental Analysis of Behavior, 1975, 23, 45-53.

Benedict, J. O., \& Ayres, J. J. B. Factors affecting conditioning in the truly random control procedure in the rat. Journal of Comparative and Physiological Psychology, 1972, 78, 323-330.

Fleshler, M., \& Hoffman, H. S. A progression for generating variable interval schedules. Journal of the Experimental Analysis of Behavior, 1962, 5, 329-330.

Hallam, R.. Rachman, S., \& Falkowski, W. Subjective, attitudinal and physiological effects of electrical aversion therapy. Behavior Research and Therapy, 1972, 10, 1-13.

Quinsey, V. L. Methodological issues in evaluating the effectiveness of aversion therapies for institutionalized child molesters. Canadian Psychologist, 1973, 14, 350-361.

SACHS, D. A., \& Keller, T. Intensity and temporal characteristics of the CER paradigm with humans. Journal of General Psychology, 1972, 86, 181-188.

Rachman, S., \& TEasdale, J. Aversion therapy and behavior disorders: An analysis. Coral Gables, Fla: University of Miami Press, 1969.

(Received for publication November 3, 1975.) 